\title{
LIFO Distortions in the Manufacturing Industry
}

\author{
June $\operatorname{Li}^{1} \&$ Megan Y. Sun ${ }^{1}$ \\ ${ }^{1}$ College of Business and Economics, University of Wisconsin, River Falls, WI 54022, USA \\ Correspondence: June Li, College of Business and Economics, University of Wisconsin, River Falls, WI 54022, \\ USA
}

Received: January 1, 2016

Accepted: January 19, 2016

Online Published: January 26, 2016

doi:10.5430/afr.v5n1p191

URL: http://dx.doi.org/10.5430/afr.v5n1p191

\begin{abstract}
For years, LIFO (Last in First Out) inventory method has been used by the U.S. companies for its tax advantages as long as LIFO is also used for financial reporting purposes (the "conformity rule"). However, LIFO is prohibited under IFRS (the International Financial Reporting Standards). With the impending acceptance of IFRS by the SEC and the Obama administration's budget proposals $(2010,2011$ and 2012) which contained a provision to eliminate LIFO for tax purposes, LIFO is expected to be repealed. This study examines the use of LIFO in the manufacturing industry from 2008 (the start of recession) through 2014 with a focus on income distortions \& liquidity measurements. This study provides transparency of LIFO accounting information in the manufacturing industry.
\end{abstract}

Keywords: LIFO, Manufacturing Industry, IFRS

\section{Introduction}

LIFO has been a very controversial inventory method. LIFO assigns the most recent prices to cost of goods sold and oldest prices to remaining inventory, hence resulting in the lowest taxable income and the highest tax savings in an inflationary period. In addition to tax savings, since LIFO allows companies to match current income with the current higher cost of inventory, industries that often experience rising inventory costs typically use LIFO as the inventory accounting method. As a result, the LIFO method enables businesses to avoid phantom profits caused by inflation.

Internal Revenue Code section 472 allows a company to use the LIFO for tax purposes if it also uses LIFO for financial reporting purposes (the "conformity rule"). The Obama administration's budget proposals (2010, 2011, and 2012) will require US publicly traded companies that currently use LIFO to change their inventory accounting method to first-in-first-out (FIFO) or average-cost as permitted under IFRS. The repeal of LIFO will cause these companies which previously use LIFO inventory valuation method to shift to FIFO and will hence significantly increase their tax liabilities. The hardest hit industries by the repeal of LIFO documented by numerous studies are the oil and manufacturing industries (e.g. Leone 2010). Much research has been conducted on the LIFO and oil industry (e.g. Li \& Sun 2014, Lirely et. al 2010 and Lirely et al. 2010). There is inadequate research on the impact of LIFO on the manufacturing industry.

To fill the void in the research, our study examines the use of LIFO in the manufacturing industry from 2008 (the start of recession) through 2014. We focus on providing transparency of LIFO information on the manufacturing industry and disclosing accounting distortion caused by the use of LIFO in manufacturing companies.

Our study is arranged as follows. In section 2, we present prior research. In section 3, we discuss data and methodology. In section 4, we provide empirical results. We conclude the paper with a summary of evidence in section 5 .

\section{Prior Research}

During an inflationary period, inventory value under LIFO is lower than its current market value. LIFO therefore grossly understates inventory values on the balance sheet and reports highest cost of goods and lowest tax liability on the income statement. In other words, LIFO creates an enhanced income statement by distorting the balance sheet. Starting from 1972, The SEC requires all publicly traded companies to report LIFO reserve. LIFO reserve is defined as the excess of current cost or replacement cost of inventory over LIFO values stated on the balance sheet. Therefore, the LIFO reserve represents the cumulative inventory value differential between LIFO and an alternative 
inventory valuation method. Hence the LIFO reserve reflects the cumulative income differential that a company could have reported over the time period while it is on LIFO.

The importance of LIFO reserve has been widely studied and reported in accounting research in recent years (Houmes and Chira 2015, Jacob and Zhang 2014, Harris and Stahlin 2014, Harris, Stahlin_and Shubita, 2014, Kostolansky and Polnaszek 2013, Reineking, et. all . 2013). Accounting research has shown that LIFO reserve could be very substantial relative to the reported inventory during increasing inventory prices. For example, Reeve and Stange (1987) find a positive relationship between the years a company has been using LIFO and the LIFO reserve. They document an LIFO reserve of about $38 \%$ of the reported LIFO inventory on 56 selected companies. Kostolansky (2009) reports that 38 percent of the Fortune 500 companies report inventory using LIFO method. They prove that LIFO causes significant differences in the reported value of inventory and net income. Coffee, Roig, Lirely, and Little (2009) study 355 active publicly traded US companies with a positive LIFO reserve and focus on accounting distortions on liquidity measures created by the use of LIFO inventory valuation method. They find significant balance sheet distortions in areas of inventory turnover, current ratio, and working capital across different company sizes and different industries. This study, however, includes data for 2007 only and focuses on many industries.

Lirely, Coffee, Roig, Swanger (2010) provide us with a good picture of the industries benefit most from using LIFO inventory valuation method. They focus on 22 energy companies that represent slightly more than 5\% of 406 energy US companies included in the Compustat North American database. While they show a limited overall use of LIFO in the energy industry, they do document a material LIFO impact for some of the LIFO users. They provide evidence that LIFO inventory valuation method produces material accounting distortions for energy companies both in absolute dollar amounts and in amounts relative to other assets and liabilities. A greater distortion is observed in the energy industry, followed by the manufacturing industry, than that in other industries.

Much research has been conducted on LIFO and the energy industry (e.g. Guenther and Sansing 2012, Coffee, et. all. 2009, Moreland 2007, Lessard 2007). More recently, Murdoch, Dehning and Krause (2013) find that the LIFO earnings is superior to FIFO earnings for predicting operating cash flows especially for the manufacturing and services industries among the 10 industries examined. However, Research on the impact of LIFO on the manufacturing industry is still limited. Our study fills this gap.

\section{Data and Methodology}

We acquire data from Compustat/ Research Insight North American database (2015). One hundred and thirty four (134) manufacturing firms were identified as LIFO users. Excluding firms with incomplete data, the final sample size is 122 . Relevant data spanning from 2008 to 2014 are obtained for all these firms.

To measure the accounting distortion, we compare inventory turnover, working capital, gross profit, and current ratio as reported in the financial statement with those adjusted with LIFO reserve. We define:

Adjusted Ending Inventory = Ending Reported Inventory + LIFO reserve

Adjusted Beginning inventory $=$ Beginning Reported Inventory + LIFO reserve from previous year

Adjusted Average Inventory $=($ Adjusted Ending Inventory + Adjusted Beginning inventory $) / 2$

Adjusted Inventory Turnover Ratio $=($ Cost of Goods sold - LIFO reserve $) /$ adjusted Average inventory

Adjusted Gross Profit $=$ Sales - Adjusted Cost of Goods Sold

Adjusted Working Capital $=$ Reported Working Capital + LIFO reserve

Adjusted Current Ratio $=($ Reported Current Asserts + LIFO Reserve $) /$ Reported Current Liability

The accounting distortion in inventory turnover ratio is the percentage difference between the adjusted inventory turnover ratio and reported inventory turnover ratio. The accounting distortion in gross profit is the percentage difference between the adjusted gross profit and the reported gross profit. Similarly, we measure the accounting distortion in working capital as the percentage difference between the adjusted working capital and reported working capital, while the accounting distortion in current ratio as the percentage difference between adjusted current ratio and reported current ratio.

\section{Empirical Results}

In a period of inflation, LIFO results in the highest amount of cost of goods sold and the lowest taxable income and thus highest tax savings compared to other inventory cost methods. As a result, for those firms using LIFO, a positive LIFO reserve is expected during rising inventory cost periods. 
Table 1. LIFO Reserve* (2008-2014) in Millions in Ranks of 2014 Amounts (Top 20 Firms \& Mean for All 122 Firms)

\begin{tabular}{lccccccc}
\hline Company Name & Y08 & Y09 & Y10 & Y11 & Y12 & Y13 & Y14 \\
\hline EXXON MOBIL CORP & 10000 & 17100 & 21300 & 25600 & 21300 & 21200 & 10600 \\
CHEVRON CORP & 9368 & 5491 & 6975 & 9025 & 9292 & 9150 & 8135 \\
CATERPILLAR INC & 3183 & 3003 & 2575 & 2422 & 2750 & 2504 & 2430 \\
TESORO CORP & 405 & 1100 & 1400 & 1700 & 1600 & 1700 & 1642 \\
DEERE \& CO & 1324 & 1367 & 1398 & 1486 & 1421 & 1529 & 1528 \\
UNITED STATES STEEL CORP & 1100 & 1100 & 885 & 1100 & 1000 & 1000 & 1000 \\
VALERO ENERGY CORP & 686 & 4500 & 6100 & 6800 & 6700 & 6900 & 857 \\
ALCOA INC & 1078 & 717 & 742 & 801 & 770 & 691 & 767 \\
IMPERIAL OIL LTD & 812 & 1509 & 1857 & 2160 & 1776 & 1680 & 739 \\
ALTRIA GROUP INC & 700 & 800 & 700 & 600 & 600 & 700 & 700 \\
DOW CHEMICAL & 627 & 818 & 1003 & 1105 & 842 & 854 & 569 \\
EASTMAN CHEMICAL CO & 525 & 446 & 490 & 590 & 505 & 506 & 462 \\
AK STEEL HOLDING CORP & 822 & 405 & 514 & 524 & 435 & 397 & 376 \\
INTL PAPER CO & 313 & 306 & 334 & 350 & 381 & 417 & 334 \\
SHERWIN-WILLIAMS CO & 321 & 250 & 277 & 379 & 357 & 337 & 332 \\
LORILLARD INC & 155 & 189 & 206 & 223 & 245 & 264 & 283 \\
KIMBERLY-CLARK CORP & 255 & 209 & 246 & 280 & 231 & 242 & 220 \\
PARKER-HANNIFIN CORP & 217 & 204 & 194 & 206 & 212 & 207 & 208 \\
REYNOLDS AMERICAN INC & 112 & 190 & 197 & 185 & 192 & 206 & 204 \\
CARPENTER TECHNOLOGY CORP & 448 & 306 & 332 & 355 & 280 & 222 & 196 \\
\hline MEAN & 312 & 372 & 439 & 511 & 465 & 461 & 297 \\
\hline
\end{tabular}

*LIFO Reserve: Year-End LIFO Reserve

Table 1 presents the total dollar amounts of the LIFO reserve for the top 20 manufacturing companies ranked according to 2014 data. Reynolds American has the lowest LIFO reserve in most of the seven years and hence the smallest dollar amount inventory accounting distortions. The Exxon Mobil has the greatest LIFO reserve in the entire seven-year period, leading to the largest potential dollar amount accounting distortions in inventory. The average LIFO reserves for all the 122 firms are also provided. We can clearly observe an increasing LIFO reserve pattern from 2008 to 2011 and a decreasing LIFO reserve starting from 2012. A closer examination reveals that almost all the companies in the manufacturing industry see a LIFO reserve reduction in recent years. 
Table 2. LIFO Reserve as A Percentage of Inventory* (2008-2014) in Ranks of 2014 Percentage (Top 20 Firms \& Mean for All 122 Firms)

\begin{tabular}{lccccccc}
\hline Company Name & Y08 & Y09 & Y10 & Y11 & Y12 & Y13 & Y14 \\
\hline CHEVRON CORP & 136.68 & 99.31 & 126.98 & 162.82 & 151.24 & 143.42 & 125.06 \\
TREX CO INC & 43.66 & 33.64 & 98.38 & 97.65 & 135.37 & 109.23 & 106.54 \\
STURM RUGER \& CO INC & 371.37 & 400.32 & 381.07 & 366.80 & 237.92 & 164.03 & 90.80 \\
IMPERIAL OIL LTD & 147.70 & 279.96 & 352.75 & 288.19 & 213.91 & 173.50 & 76.45 \\
UNITED REFINING CO & 161.92 & 2.22 & 24.30 & 53.56 & 49.75 & 83.22 & 72.43 \\
LORILLARD INC & 60.78 & 67.26 & 74.37 & 80.51 & 59.76 & 52.91 & 70.05 \\
ENCORE WIRE CORP & 35.27 & 139.59 & 206.20 & 98.86 & 115.33 & 88.15 & 68.01 \\
TESORO CORP & 51.46 & 176.85 & 111.38 & 96.43 & 101.39 & 66.28 & 67.32 \\
EXXON MOBIL CORP & 85.87 & 148.01 & 164.15 & 170.39 & 146.47 & 131.39 & 63.56 \\
GORMAN-RUPP CO & 87.54 & 117.51 & 91.55 & 69.27 & 58.53 & 61.48 & 61.10 \\
AEP INDUSTRIES INC & 40.63 & 15.85 & 30.28 & 31.53 & 32.75 & 42.29 & 51.73 \\
IKONICS CORP & 40.59 & 43.29 & 45.18 & 54.27 & 46.51 & 48.85 & 49.98 \\
BURNHAM HOLDINGS INC & 40.64 & 43.34 & 48.26 & 46.97 & 48.01 & 48.28 & 45.50 \\
FARMER BROS CO & 50.08 & 39.38 & 33.23 & 87.22 & 63.78 & 58.34 & 44.28 \\
JOHN BEAN TECHNOLOGIES & 35.37 & 43.27 & 41.89 & 37.61 & 44.60 & 41.84 & 43.02 \\
AMPCO-PITTSBURGH CORP & 33.02 & 21.91 & 32.01 & 43.33 & 39.50 & 41.17 & 42.58 \\
STARRETT (L.S.) CO -CL A & 44.97 & 55.96 & 54.60 & 44.52 & 39.35 & 54.56 & 41.67 \\
UNITED STATES STEEL CORP & 44.14 & 65.52 & 37.63 & 39.64 & 39.95 & 37.20 & 40.06 \\
GENCOR INDUSTRIES INC & 16.82 & 17.68 & 22.83 & 25.59 & 32.49 & 36.39 & 39.04 \\
NEWMARKET CORP & 28.35 & 21.25 & 17.93 & 21.19 & 30.37 & 35.45 & 38.46 \\
DEERE \& CO & 43.53 & 57.02 & 45.64 & 34.00 & 27.49 & 30.98 & 36.30 \\
\hline MEAN & 37 & 46 & 50 & 50 & 43 & 43 & 27 \\
\hline
\end{tabular}

* LIFO Reserve As A Percentage Of Inventory = LIFO Reserve / Reported Ending Inventory

Table 2 depicts the results of the LIFO reserve as a percentage of inventory. LIFO reserve as a percentage of inventory measures accounting inventory distortion. Sturm Ruger \& Co has the greatest LIFO reserve to inventory percentage in most of the seven years and therefore the greatest accounting inventory distortion. The percentage and the accounting inventory distortion decreased starting from 2012. The average distortion in inventory had a steady increase from $37 \%$ to $50 \%$ for 2008 through 2011 . The distortion percentage, however, decreased from $43 \%$ to $27 \%$ for 2012 to 2014. Similar to observations derived from LIFO reserve amounts in Table 1, the distortion increased in early recession years and declined in recent years. 
Table 3. Lifo Reserve as A Percentage of Net Sales* (2008-2014) in Ranks of 2014 Percentage (Top 20 Firms \& Mean for All 122 Firms)

\begin{tabular}{lccccccc}
\hline Company Name & Y08 & Y09 & Y10 & Y11 & Y12 & Y13 & Y14 \\
\hline GENCOR INDUSTRIES INC & 6.7 & 8.3 & 7.4 & 7.4 & 7.9 & 10.5 & 13.7 \\
GORMAN-RUPP CO & 15.1 & 17.9 & 15.9 & 14.1 & 14.2 & 14.1 & 13.3 \\
STARRETT (L.S.) CO -CL A & 11.3 & 16.5 & 12.4 & 10.7 & 10.6 & 12.6 & 11.1 \\
BURNHAM HOLDINGS INC & 8.2 & 9.2 & 9.3 & 9.8 & 9.5 & 10.5 & 10.1 \\
CARPENTER TECHNOLOGY CORP & 22.9 & 22.4 & 27.7 & 21.2 & 13.8 & 9.8 & 9.0 \\
AMPCO-PITTSBURGH CORP & 5.2 & 5.1 & 6.7 & 8.6 & 9.5 & 9.4 & 8.5 \\
STURM RUGER \& CO INC & 24.4 & 14.2 & 14.6 & 11.4 & 7.7 & 5.5 & 7.4 \\
IKONICS CORP & 5.4 & 5.9 & 6.0 & 7.2 & 7.2 & 7.1 & 7.1 \\
ALLIED HEALTHCARE PRODS INC & 4.3 & 4.3 & 5.2 & 5.6 & 5.8 & 6.1 & 6.8 \\
TREX CO INC & 9.2 & 5.6 & 9.0 & 10.6 & 7.7 & 7.1 & 6.5 \\
FARMER BROS CO & 10.2 & 7.9 & 6.2 & 15.0 & 8.5 & 7.0 & 6.0 \\
AK STEEL HOLDING CORP & 10.8 & 9.9 & 8.6 & 8.1 & 7.3 & 7.1 & 5.8 \\
NEWMARKET CORP & 3.5 & 2.7 & 2.7 & 3.0 & 4.4 & 4.8 & 5.7 \\
UNITED STATES STEEL CORP & 4.6 & 10.0 & 5.1 & 5.5 & 5.2 & 5.7 & 5.7 \\
LORILLARD INC & 4.4 & 5.1 & 5.1 & 5.0 & 5.3 & 5.3 & 5.6 \\
KOSS CORP & 2.6 & 1.9 & 2.0 & 2.3 & 3.1 & 3.7 & 4.9 \\
EASTERN CO & 4.6 & 4.2 & 4.0 & 4.4 & 4.0 & 4.7 & 4.9 \\
JOHN BEAN TECHNOLOGIES & 4.2 & 5.5 & 5.1 & 4.8 & 5.3 & 5.3 & 4.9 \\
EASTMAN CHEMICAL CO & 7.8 & 8.8 & 8.4 & 8.2 & 6.2 & 5.4 & 4.8 \\
MATERION CORP & 8.2 & 9.4 & 6.2 & 6.1 & 7.1 & 6.6 & 4.8 \\
\hline MEAN & 2.4 & 4.1 & 4.0 & 3.8 & 3.4 & 3.5 & 2.3 \\
\hline
\end{tabular}

*LIFO Reserve As A Percentage Of Net Sales = LIFO Reserve/ Ending Net Sales

Table 3 presents LIFO reserve as a percentage of net sales. LIFO reserve as a percentage of net sales is another important measure of accounting distortion. Carpenter Technology is shown to have the greatest percentage of LIFO reserve to net sales in most years. On average, LIFO reserve as a percentage of net sales peaked at $4.1 \%$ in 2009 and steadily decreased from 2010 to $2.3 \%$ in 2014. Similar to prior observations, accounting distortion in terms of LIFO reserve as a percentage of net sales subsided in recent years. 
Table 4. LIFO Inventory Distortion* (2008-2014) in Ranks of 2014 Percentage (Top 20 Firms \& Mean for All 122 Firms)

\begin{tabular}{lccccccc}
\hline Company Name & Y08 & Y09 & Y10 & Y11 & Y12 & Y13 & Y14 \\
\hline CHEVRON CORP & 136.7 & 99.3 & 127.0 & 162.8 & 151.2 & 143.4 & 125.1 \\
TREX CO INC & 43.7 & 33.6 & 98.4 & 97.7 & 135.4 & 109.2 & 106.5 \\
STURM RUGER \& CO INC & 371.4 & 400.3 & 381.1 & 366.8 & 237.9 & 164.0 & 90.8 \\
IMPERIAL OIL LTD & 147.7 & 280.0 & 352.8 & 288.2 & 213.9 & 173.5 & 76.4 \\
UNITED REFINING CO & 161.9 & 2.2 & 24.3 & 53.6 & 49.8 & 83.2 & 72.4 \\
LORILLARD INC & 60.8 & 67.3 & 74.4 & 80.5 & 59.8 & 52.9 & 70.0 \\
ENCORE WIRE CORP & 35.3 & 139.6 & 206.2 & 98.9 & 115.3 & 88.1 & 68.0 \\
TESORO CORP & 51.5 & 176.8 & 111.4 & 96.4 & 101.4 & 66.3 & 67.3 \\
EXXON MOBIL CORP & 85.9 & 148.0 & 164.1 & 170.4 & 146.5 & 131.4 & 63.6 \\
GORMAN-RUPP CO & 87.5 & 117.5 & 91.5 & 69.3 & 58.5 & 61.5 & 61.1 \\
AEP INDUSTRIES INC & 40.6 & 15.8 & 30.3 & 31.5 & 32.7 & 42.3 & 51.7 \\
IKONICS CORP & 40.6 & 43.3 & 45.2 & 54.3 & 46.5 & 48.8 & 50.0 \\
BURNHAM HOLDINGS INC & 40.6 & 43.3 & 48.3 & 47.0 & 48.0 & 48.3 & 45.5 \\
FARMER BROS CO & 50.1 & 39.4 & 33.2 & 87.2 & 63.8 & 58.3 & 44.3 \\
JOHN BEAN TECHNOLOGIES & 35.4 & 43.3 & 41.9 & 37.6 & 44.6 & 41.8 & 43.0 \\
AMPCO-PITTSBURGH CORP & 33.0 & 21.9 & 32.0 & 43.3 & 39.5 & 41.2 & 42.6 \\
STARRETT (L.S.) CO -CL A & 45.0 & 56.0 & 54.6 & 44.5 & 39.4 & 54.6 & 41.7 \\
UNITED STATES STEEL CORP & 44.1 & 65.5 & 37.6 & 39.6 & 40.0 & 37.2 & 40.1 \\
GENCOR INDUSTRIES INC & 16.8 & 17.7 & 22.8 & 25.6 & 32.5 & 36.4 & 39.0 \\
NEWMARKET CORP & 28.3 & 21.3 & 17.9 & 21.2 & 30.4 & 35.4 & 38.5 \\
\hline MEAN & 36.9 & 46.2 & 50.1 & 50.1 & 42.6 & 42.8 & 27.2 \\
\hline
\end{tabular}

*Inventory Distortion = (Adjusted Ending Inventory - Reported Ending Inventory $) /$ Reported Ending Inventory

Tables 4 to 8 provide more insights into liquidity measures. Table 4 reports the LIFO inventory distortion percentage, where we compare the inventory valued under LIFO with inventory valued using current costs. LIFO inventory distortion percentage measures balance sheet accounting distortion created by LIFO. Sturm Ruger \& Co has the greatest distortion in the early years but has significantly reduced in recent years. Consistent with prior observations, LIFO inventory distortion peaked in $2010 / 2011(50.1 \%)$ and the trend reversed in recent years with the lowest level in 2014 (27.2\%). 
Table 5. LIFO Inventory Turnover Distortion* (2008-2014) in Ranks of 2014 Percentage (Top 20 Firms \& Mean for All 122 Firms)

\begin{tabular}{lccccccc}
\hline Company Name & Y08 & Y09 & Y10 & Y11 & Y12 & Y13 & Y14 \\
\hline CHEVRON CORP & -59.3 & -56.5 & -55.3 & -61.2 & -63.2 & -61.8 & -59.6 \\
STURM RUGER \& CO INC & -85.5 & -83.9 & -84.3 & -82.8 & -77.5 & -69.2 & -59.2 \\
TREX CO INC & -37.0 & -34.9 & -45.8 & -57.2 & -58.2 & -59.6 & -57.0 \\
IMPERIAL OIL LTD & -72.5 & -71.2 & -78.3 & -78.2 & -73.6 & -68.2 & -56.9 \\
EXXON MOBIL CORP & -62.1 & -57.5 & -64.1 & -65.4 & -63.8 & -60.9 & -51.0 \\
GORMAN-RUPP CO & -57.2 & -62.2 & -61.8 & -55.0 & -50.8 & -49.8 & -49.5 \\
ENCORE WIRE CORP & -41.4 & -49.0 & -67.2 & -61.2 & -55.4 & -53.3 & -46.6 \\
LORILLARD INC & -43.6 & -45.7 & -47.9 & -49.8 & -47.2 & -43.7 & -45.7 \\
UNITED REFINING CO & -48.1 & -32.5 & -12.9 & -29.6 & -35.7 & -41.5 & -45.6 \\
STARRETT (L.S.) CO -CL A & -44.1 & -50.2 & -48.0 & -44.4 & -40.7 & -44.6 & -44.1 \\
TESORO CORP & -48.4 & -55.0 & -60.2 & -53.6 & -52.4 & -47.0 & -42.7 \\
BURNHAM HOLDINGS INC & -34.8 & -38.3 & -39.9 & -41.1 & -41.0 & -42.1 & -41.2 \\
IKONICS CORP & -32.1 & -36.9 & -38.1 & -41.7 & -41.8 & -40.8 & -41.0 \\
FARMER BROS CO & -45.1 & -42.1 & -35.5 & -53.2 & -51.6 & -45.7 & -40.5 \\
GENCOR INDUSTRIES INC & -21.5 & -23.3 & -24.0 & -26.7 & -30.1 & -36.0 & -40.3 \\
VALERO ENERGY CORP & -44.2 & -39.8 & -55.7 & -57.5 & -56.1 & -56.1 & -38.9 \\
AMPCO-PITTSBURGH CORP & -26.5 & -27.3 & -28.8 & -35.8 & -38.0 & -37.4 & -37.0 \\
AK STEEL HOLDING CORP & -58.3 & -60.3 & -55.9 & -58.3 & -52.2 & -45.7 & -34.9 \\
AEP INDUSTRIES INC & -28.2 & -23.9 & -21.4 & -26.7 & -26.8 & -30.4 & -34.8 \\
JOHN BEAN TECHNOLOGIES & -28.1 & -33.7 & -34.9 & -33.1 & -34.2 & -35.2 & -34.6 \\
\hline MEAN & -30.8 & -28.7 & -30.5 & -31.1 & -28.8 & -26.7 & -22.9 \\
\hline
\end{tabular}

* Inventory Turnover Distortion =(Adjusted Inventory Turnover-Reported Inventory Turnover)/Reported Inventory Turnover

Table 5 depicts how inventory turnover ratio in the 122 manufacturing companies is distorted by the use of the LIFO. Sturm Ruger \& Co has the greatest distortion in inventory turnover ratio in most of the seven-year period. The average inventory turnover distortions were $-30.8 \%$ to $-31.1 \%$ from 2008 through 2011. From 2012, the average inventory turnover distortions started to decline to $-22.9 \%$ in 2014 . Despite the reversal of the distortion in inventory turnover ratio, the distortion remained to be significant. 
Table 6. LIFO Gross Profit Distortion* (2008-2014) in Ranks of 2014 Percentage (Top 20 Firms \& Mean for All 122 Firms)

\begin{tabular}{lccccccc}
\hline Company Name & Y08 & Y09 & Y10 & Y11 & Y12 & Y13 & Y14 \\
\hline TESORO CORP & 36.4 & 205.6 & 216.4 & 104.5 & 69.9 & 117.6 & 69.3 \\
GENCOR INDUSTRIES INC & 26.7 & 46.6 & 40.7 & 43.0 & 38.1 & 42.0 & 59.5 \\
AK STEEL HOLDING CORP & 166.8 & 123.8 & 148.3 & 250.6 & 159.6 & 71.6 & 59.3 \\
UNITED STATES STEEL CORP & 26.4 & -185.2 & 79.4 & 70.5 & 58.3 & 71.0 & 48.7 \\
GORMAN-RUPP CO & 58.6 & 67.3 & 54.2 & 50.0 & 50.6 & 50.3 & 47.4 \\
AMPCO-PITTSBURGH CORP & 18.1 & 15.9 & 22.6 & 33.5 & 41.7 & 41.4 & 42.9 \\
BURNHAM HOLDINGS INC & 33.8 & 35.4 & 37.4 & 39.3 & 36.2 & 40.1 & 38.5 \\
ENCORE WIRE CORP & 16.8 & 93.1 & 90.3 & 40.6 & 70.2 & 41.6 & 37.9 \\
CARPENTER TECHNOLOGY CORP & 88.7 & 118.6 & 162.8 & 112.6 & 57.5 & 41.1 & 37.7 \\
UNITED REFINING CO & 140.8 & 2.1 & 53.2 & 34.9 & 13.8 & 21.0 & 32.4 \\
CONTINENTAL MATERIALS CORP & 26.8 & 23.3 & 22.5 & 28.5 & 34.4 & 30.9 & 32.2 \\
STARRETT (L.S.) CO -CL A & 31.6 & 48.5 & 34.9 & 28.4 & 31.2 & 37.8 & 30.2 \\
AEP INDUSTRIES INC & 28.7 & 6.5 & 16.9 & 21.6 & 15.2 & 22.4 & 29.1 \\
WINNEBAGO INDUSTRIES & 83.5 & -132.4 & 90.7 & 69.9 & 63.9 & 33.4 & 29.0 \\
ALLIED HEALTHCARE PRODS INC & 16.5 & 17.0 & 19.2 & 21.1 & 22.4 & 24.5 & 28.8 \\
KATY INDUSTRIES INC & 21.0 & 12.9 & 17.1 & 28.0 & 26.9 & 31.3 & 27.2 \\
KOPPERS HOLDINGS INC & 18.9 & 25.2 & 23.3 & 21.5 & 22.0 & 23.3 & 25.2 \\
MATERION CORP & 40.5 & 56.8 & 32.1 & 36.7 & 38.1 & 34.3 & 22.0 \\
STURM RUGER \& CO INC & 92.1 & 40.5 & 40.0 & 30.0 & 19.6 & 13.6 & 19.7 \\
EASTERN CO & 21.0 & 19.1 & 17.1 & 20.2 & 17.1 & 19.7 & 19.1 \\
\hline MEAN & 10.3 & 16.1 & 15.2 & 15.2 & 13.7 & 14.2 & 9.3 \\
\hline
\end{tabular}

*Gross Profit Distortion= (Adjusted Gross Profit - Reported Gross Profit)/Reported Gross Profit

Table 6 documents gross profit distortion. The accounting distortion in gross profit is the percentage difference between the adjusted gross profit and the reported gross profit. AK Steel Holding is found to have the greatest gross profit distortion in 2008, 2011, and 2012, while Tesoro Corp has the greatest gross profit distortion in 2009, 2010, 2013 and 2014. Following the similar pattern in prior observations, the average gross profit distortion peaked in 2009 at $16.1 \%$ and declined to $9.3 \%$ in 2014 . As in prior observations, the levels of gross profit distortion continued to be significant. 
Table 7. LIFO Working Capital Distortion* (2008-2014) in Ranks of 2014 Percentage (Top 20 Firms \& Mean for All $\underline{122 \text { Firms) }}$

\begin{tabular}{lccccccc}
\hline Company Name & Y08 & Y09 & Y10 & Y11 & Y12 & Y13 & Y14 \\
\hline WAUSAU PAPER CORP & 43.1 & 51.8 & 49.7 & 78.5 & 66.5 & 26.8 & 1382.9 \\
TESORO CORP & 197.6 & 329.3 & 324.1 & 188.5 & 91.2 & 88.6 & 102.1 \\
CHEVRON CORP & 210.7 & 49.9 & 35.2 & 46.0 & 43.2 & 53.1 & 78.9 \\
TREX CO INC & 46.6 & 25.2 & 43.2 & -151.9 & 233.5 & 84.5 & 70.7 \\
STURM RUGER \& CO INC & 95.9 & 59.1 & 52.1 & 38.8 & 101.8 & 54.9 & 70.2 \\
HERSHEY CO & 184.4 & 39.5 & 21.7 & 19.1 & 31.0 & 15.3 & 55.3 \\
POTLATCH CORP & -12.8 & 14.7 & 8.9 & 16.9 & 14.2 & 14.1 & 51.2 \\
JOHN BEAN TECHNOLOGIES & 47.0 & 66.3 & 39.1 & 38.5 & 24.3 & 41.3 & 47.4 \\
AEP INDUSTRIES INC & 30.0 & 15.7 & 31.3 & 27.7 & 32.3 & 34.6 & 43.3 \\
GORMAN-RUPP CO & 50.4 & 54.0 & 56.4 & 48.3 & 48.0 & 43.0 & 42.5 \\
AK STEEL HOLDING CORP & 64.8 & 45.6 & 91.9 & 381.6 & 69.0 & 89.7 & 41.7 \\
BURNHAM HOLDINGS INC & 35.8 & 36.5 & 43.6 & 44.6 & 48.0 & 50.0 & 40.4 \\
EASTMAN CHEMICAL CO & 88.8 & 47.7 & 50.2 & 49.7 & 41.1 & 36.9 & 40.1 \\
FARMER BROS CO & 14.4 & 24.7 & 30.4 & 128.9 & 97.5 & 56.2 & 39.0 \\
UNITED REFINING CO & 74.1 & 2.3 & 30.6 & 49.3 & 24.7 & 29.1 & 37.5 \\
LENNOX INTERNATIONAL INC & 21.1 & 30.0 & 21.4 & 22.4 & 21.7 & 38.9 & 35.1 \\
UNITED STATES STEEL CORP & 37.2 & 43.3 & 41.0 & 51.8 & 41.9 & 35.3 & 34.9 \\
AIR PRODUCTS \& CHEMICALS INC & 10.6 & 13.7 & 9.0 & 11.3 & 12.1 & 44.8 & 31.8 \\
CONTINENTAL MATERIALS CORP & 19.8 & 17.5 & 20.2 & 18.0 & 21.4 & 21.0 & 29.3 \\
HARSCO CORP & 10.3 & 5.8 & 6.8 & 7.6 & 6.6 & 12.2 & 28.4 \\
\hline MEAN & 32.8 & 34.2 & 36.0 & 40.5 & 31.9 & 33.8 & 23.6 \\
\hline & & & & & & &
\end{tabular}

* Working Capital Distortion $=($ Adjusted Working Capital - Reported Working Capital $) /$ Reported Working Capital

Table 7 reports the data for the working capital distortion. The accounting distortion in working capital is calculated as the percentage difference between the adjusted working capital and reported working capital. Data show the distortion is significant in each year. Chevron Corp is found to have the greatest working capital distortion in 2008, while TESORO has the greatest distortion in 2009 and 2010. AK STEEL has an extremely high working capital distortion in 2011, while Trex Co has a similar situation in 2012. The average working capital distortion follows the trend in previous distortion measures. 2011 has the highest distortion level of $40.5 \%$ and declined to the lowest level in 2014 at $23.6 \%$. 
Table 8. LIFO Current Ratio Distortion*(2008-2014) in Ranks of 2014 Percentage (Top 20 Firms \& Mean for All $\underline{122 \text { Firms) }}$

\begin{tabular}{lccccccc}
\hline Company Name & Y08 & Y09 & Y10 & Y11 & Y12 & Y13 & Y14 \\
\hline & & & & & & & \\
STURM RUGER \& CO INC & 58.5 & 39.3 & 35.6 & 25.6 & 39.3 & 24.2 & 34.3 \\
TESORO CORP & 24.6 & 49.5 & 47.8 & 41.0 & 34.5 & 31.9 & 32.4 \\
TREX CO INC & 26.0 & 14.4 & 25.4 & 27.7 & 45.1 & 32.2 & 29.7 \\
GORMAN-RUPP CO & 37.1 & 36.2 & 32.9 & 32.5 & 30.3 & 29.2 & 28.8 \\
BURNHAM HOLDINGS INC & 22.5 & 24.2 & 26.0 & 27.4 & 26.1 & 27.7 & 27.3 \\
UNITED REFINING CO & 49.7 & 1.4 & 14.1 & 25.7 & 17.9 & 22.8 & 25.5 \\
IMPERIAL OIL LTD & 21.4 & 45.0 & 52.5 & 43.2 & 43.2 & 41.7 & 24.0 \\
AEP INDUSTRIES INC & 15.4 & 6.9 & 13.2 & 14.2 & 14.6 & 17.5 & 20.7 \\
EXXON MOBIL CORP & 13.8 & 31.0 & 36.1 & 35.1 & 33.0 & 35.7 & 20.0 \\
FARMER BROS CO & 12.5 & 14.6 & 14.6 & 44.2 & 31.0 & 25.4 & 20.0 \\
CHEVRON CORP & 25.7 & 14.8 & 14.3 & 17.0 & 16.7 & 18.2 & 19.3 \\
STARRETT (L.S.) CO -CL A & 19.8 & 29.9 & 22.8 & 18.2 & 18.2 & 23.0 & 18.6 \\
AK STEEL HOLDING CORP & 41.1 & 24.9 & 36.6 & 41.1 & 30.2 & 31.1 & 18.5 \\
KATY INDUSTRIES INC & 11.3 & 11.2 & 11.6 & 13.3 & 17.6 & 23.4 & 17.0 \\
NEWMARKET CORP & 12.8 & 6.8 & 8.1 & 9.6 & 13.3 & 12.1 & 16.8 \\
ALLIED HEALTHCARE PRODS INC & 9.6 & 10.1 & 10.5 & 11.5 & 12.2 & 13.2 & 16.6 \\
CARPENTER TECHNOLOGY CORP & 46.3 & 40.8 & 40.5 & 30.7 & 22.4 & 17.4 & 16.4 \\
UNITED STATES STEEL CORP & 19.2 & 21.9 & 16.7 & 19.1 & 18.6 & 16.5 & 15.5 \\
ENCORE WIRE CORP & 5.6 & 14.6 & 25.4 & 16.6 & 23.9 & 18.9 & 15.4 \\
IKONICS CORP & 15.4 & 14.0 & 12.7 & 14.4 & 16.8 & 15.5 & 14.8 \\
\hline MEAN & 10.4 & 9.2 & 9.5 & 9.4 & 8.9 & 8.4 & 7.4 \\
\hline & & & & & & &
\end{tabular}

*Current Ratio Distortion $=($ Adjusted Current Ratio - Reported Current Ratio $) /$ Reported Current Ratio

Table 8 reports the current ratio distortion, which is measured as the percentage difference between adjusted current ratio and reported current ratio. Sturm Ruger \& Co has the greatest current ratio distortion in 2008, while Tesoro tops all other firms in 2009 and 2010. Farmer Bros tops the list in current ratio distortion in 2011, while Imperial Oil has the greatest current ratio distortion in 2012. The average current ratio distortion was the highest at $10.4 \%$ in 2008 . The overall distortion appeared to decrease through 2014 at 7.4\%. Compared to other distortion measures, current ratio is least distorted. Consistent with all other distortion measures, current ratio distortion declined over the years.

\section{Conclusions}

Our study documents that the use of the LIFO inventory accounting method leads to significant accounting information distortions in inventory turnover, gross profit, working capital, and current ratio for the manufacturing industry. The greatest accounting distortion resulting from using LIFO is in working capital, followed by inventory turnover distortion. Although gross profit and current ratio are less distorted, the levels are significant. In addition, we observe a clear pattern of increasing accounting information distortion in the years right after recession starts. While remaining significant, the distortions start to decrease after 2011. This reversal in distortion trend helps to bolster the repeal of LIFO. Manufacturing firms appear to have gradually change their accounting inventory practices in anticipation of the adoption of IFRS under which LIFO is prohibited. We expect that the repeal of LIFO will lead to greater transparency in financial reporting in the manufacturing industry. The limitation of this paper is 
the narrow focus on the impact of LIFO in the manufacturing Industry. The observations derived might not be applicable to other industries. Future research could examine LIFO distortions in other industries.

\section{References}

Coffee, D., R. Roig, R. Lirely, \& P. Little (2009). The materiality of LIFO accounting distortion on liquidity measurements. Journal of Finance and Accountancy (January), 35-46.

Guenther, D. A. \& Sansing, R. C. (2012). Unintended consequences of LIFO repeal: the case of the oil industry. The Accounting Review, 87 (5), 1589-1602. http://dx.doi.org/10.2308/accr-50194

Kostolansky, J. (2009). The impact of LIFO in the Fortune 500 in 2007. Journal of Applied Business Research, 25(5), 11-20. http://dx.doi.org/10.19030/jabr.v25i5.1002

Kostolansky, J. \& Polnaszek, E. (2013). New perspectives on the use of LIFO and firm size. Journal of Applied Business Research, 29 (5), 1501-1508. http://dx.doi.org/10.19030/jabr.v29i5.8031

Harris, P. \& Stahlin, W. (2014). The theoretical defenses of the last in first out inventory methods. Journal of Business \& Economics Research, 12(1), 43-46. http://dx.doi.org/10.19030/jber.v12i1.8376

Harris, P., Stahlin, W. \& Shubita, M. F. (2014). US GAAP conversion to IFRS: a case study of the cash flow statement. Journal of Business Case Studies, 10(1), 15-20. http://dx.doi.org/10.19030/jbcs.v10i1.8325.

Houmes, R. \& Chira, I. (2015). The valuation effect of LIFO's repeal on high pricing power firms. Review of Accounting \& Finance, 14(3), 306-323. http://dx.doi.org/10.1108/RAF-06-2014-0062

Leone, M. (2010). Sucking the LIFO out of inventory: The government sees billions of dollars in potential tax revenue sitting on the shelves of company warehouses. CFO Magazine (July/August), 27-29.

Lessard, S. C. (2007). Giving life to LIFO: adoption of the LIFO method of inventory valuation by the income tax code. The Tax Lawyer, 60(3), 781-806.

Li, J. \& Sun, M. Y. (2014). LIFO and accounting distortion - the case of the oil industry. Journal of Accounting and Finance, 14 (5), 86-96.

Lirely, R., Coffee, D., Roig,R., \& Swanger S. (2010). LIFO accounting and liquidity measurements in the energy industry. Oil, Gas, and Energy Quarterly (No. 3), 393-406.

Moreland, K. A. (2007). International financial reporting-inventory costing at Exxon and BP. Issues in Accounting Education, 22(4), 549-561. http://dx.doi.org/10.2308/iace.2007.22.4.549

Murdoch, B., Dehning, B., \& Krause, P. (2013). Further evidence on the ability of FIFO and LIFO earnings to predict operating cash flows: An Industry Specific Analysis. The Journal of Applied Business Research, 29 (4), 1231-1241. http://dx.doi.org/10.19030/jabr.v29i4.7929

Reeve, J. M. \& Stanga K. G. (1987). Balance sheet impact of using LIFO: an empirical study. Accounting Horizons (September), 9-15.

Reineking, C., Chamberlain, D.H., Rudolph, H. \& Smith M. (2013). An examination of inventory costing convergence under Generally Accepted Accounting Principles and International Financial Reporting Standards. Journal of International Business Research, 17-32.

Thomas, J. \& Zhang, F. (2014). Valuation of tax expense. Review of Accounting Studies, 19 (4), 1436-1467. http://dx.doi.org/10.1007/s11142-013-9274-3 